$\xi=-1$

\title{
Optimal approach for building scheduling algorithm in satellite communication
}

\author{
Ali M. Al-Saegh \\ Computer Engineering Techniques, Almamon university College, Baghdad, Iraq \\ Email: ali.alsaegh84@gmail.com
}

\begin{abstract}
Building scheduling algorithms in satellite communication links became a necessity according to the typical problems that satellite networks suffers from, such as congestions, jamming, mobility, atmospheric impairment, and achieving the quality of service (QoS) requirements. However, building efficient algorithms needs several considerations that should be taken into account. Such as satellite and earth station node(s), link parameters and specifications, along with the service requirements and limitations. This paper presents efficient approach for accumulating the effective considerations that the designer should employ as a framework for building proper and efficient scheduling algorithm. The proposed approach provides proper solutions to the satellite communications impairments and satisfies the quality of service requirements in satellite communication networks.
\end{abstract}

Keywords: Performance matrices; Quality of Service QoS; Resource management; Satellite communications; Scheduling algorithm.

\section{Introduction}

The worldwide development of the satellite networks industry as well as developing the research departments around the world has turned into an essential issue at the moment in view of the fact that recently there has been a sensible requirement for higher bandwidths and long-distance communication link. As a result of this, network requirements and facilities are required to be boosted by the service providers in satellite communications who are also expected to enhancing the performance as well as managing the resources.

To this end, there is also a need in servicing larger areas via satellite networks and multi-service type demands. Furthermore, the researchers have begun optimizing the channel features alongside managing the link resources and developing the mitigation techniques. To get accustomed to such a trend, satellite service providers' profession is to improve the communication platforms as well as provide managed products and services, which are being regarded as fundamental constituents of critical business operations. It is to be noted here that the most considerable issue with the area communication which has numerous ground stations is the problems appears in sharing the available bandwidth among the ground stations in a manner that reaches significant satellite utilization. However, the amount and type of the traffic between each pair of the ground stations will be commonly defined by an available traffic matrix. Apparently, only one ground station is responsible for transmitting each transponder's frequency any time. In addition, the ground stations are also responsible for identifying the data to be received and from which particular transponder. In order to fulfill such a demand, a schedule is needed to be determined for defining the order in which that data is required to be transmitted as well as defining which transponder should do so.

However, substantial problems had been appeared through time, especially with the development of the high quality satellite ser- vices and applications. Such as atmospheric impairment, mobility, jamming, congestions. Moreover, many problems appeared in sharing the available bandwidth among the ground stations, along with the problems that appeared due to unbalanced traffic maps that happened because of the multi-destination transmissions and discreteness of data sizes.

These aforementioned problems raise the demand to specify an efficient approach for building appropriate scheduling algorithm to find a proper solution to solve or decrease the effects of these problems. The approach takes into account the performance matrices, user requirements, protocol stacks, service limitations, connection environment and obstacles, control factors, and link specifications.

Consequently, the methodology of the proposed approach is introduced in section 2 with full specifications needed to be characterized in the approach starting from the wider field consideration then narrowing down the specifications concerning the satellite and earth stations as terminals, along with the quality of service requirements, layer specifications, and scheduling techniques; before concluding the outcomes in section 3 .

\section{Methodology}

Due to the fact that the ground stations entail transmitting diverse amounts of data for different destinations, which yields rather unbalanced traffic maps, the abovementioned scheduling problem seems more complicated, accordingly. Such a problem can be even exacerbated by multi-destination transmissions and discreteness of data sizes. In order to reduce the time a transponder being idle, a noticeable attention should be granted on organizing such a schedule. It needs to be asserted that the idle time emerges customarily for the reason that a pair of ground stations has the data to communicate and a non-busy transponder is available, nonetheless, one or both ground stations are by this time supposed to 
communicate with another ground station via another transponder while at this moment, there is no other traffic available for the idle transponder.

It is to be highlighted that frequency agility is a proper alternative for enhancing the satellite bandwidth utilization; nevertheless, few networks implement such an alternative for their earth stations; such avoidance is more popular with those networks which have unbalanced or centralized traffic, like the corporate star networks. Ground stations, which have light transmission traffic, are normally able to transmit and receive data only from one transponder. Though, the scheduler might be seriously constrained by such a cost reduction and might be influenced adversely to the satellite utilization as a result.

The QoS levels provided by a network can be remarkably influenced by the nature of scheduling mechanisms used on network links. Realistically, arbitrating between the packets that are prepared to be transmitted on the link is considered as the rudimentary function of the scheduler. Accordingly, particular performance measures can be attained considering the scheduling algorithm and the traffic characteristics of the flows multiplexed on the link. To eventually deliver end-to-end QoS guarantees, these can be employed by the network, consequently.

In order to build a scheduling algorithm for satellite communication system, several specifications needed to be characterized starting from the wider field consideration then narrowing down the specifications. However, the general framework should be drawn prior to the commencement of the aforementioned process This framework consists of the transmitter earth station(s), as well as the Satellite(s) and receiver earth stations(s) as shown in fig. 1.

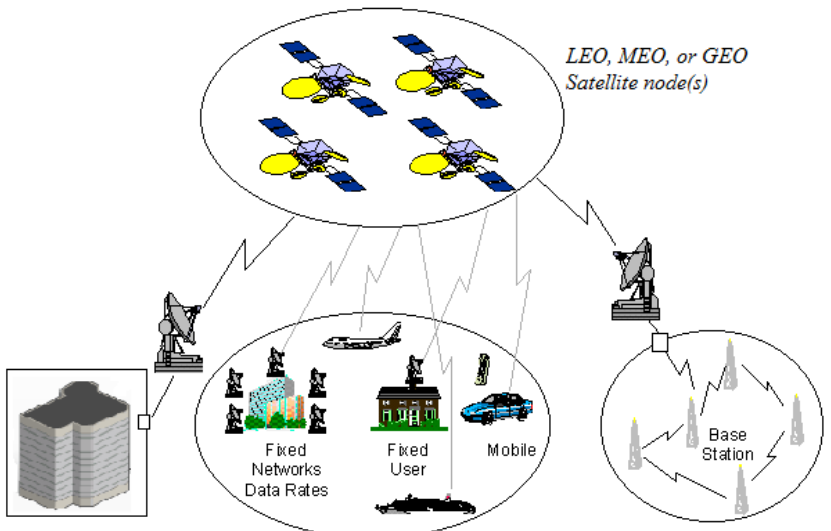

Fig. 1: Satellite communication general framework.

Since particular parts from this framework should be determined to be elaborated on, single or multi earth stations can be employed as a transmitter while the satellite is as a receiver, satellite(s) as a transmitter and earth station(s) as a receiver, or from earth station(s) to another earth station(s) via satellite as a double hop link. It can be declared that the transmission direction plays a significant role while the scheduling algorithm is intended to be constructed that might be either uplink or downlink, or sometimes the algorithm contains both.

We suppose that the link framework entails a single satellite for simplicity as well as reducing the conflict in the coming subsections. In addition, we should determine the specifications to both types of nodes, Satellite and earth station(s) nodes for constructing the algorithm.

\subsection{Satellite node specifications}

During the designing process of the specific framework, the type of satellite should be selected according to the orbit type. There exist three types of satellite orbits, namely the Geostationary Earth Orbit (GEO), Medium Earth Orbit (MEO), and Low Earth orbit (LEO). The former appears stationary with respect to the earth station with hop length of approximately $36000 \mathrm{~km}$ so that; the satellite mobility should not be considered, consequently. MEO and LEO have lesser hop length, and it appears a continually moving communication node with respect to the earth station(s); henceforth, the mobility should be considered.

After considering the satellite type according to its orbit, we should now specify its type according to the transmission link type which is either Unicast, Multicast, or Broadcast [1,2]. The former deals with a connection between the satellite and a single user (point to point), as several military or special application types of connections, and it can be used in uplink and downlink. On the other hand, the Multicast is the connection between the satellite and a specific group of users, and Broadcasting is the process of sending the data to all the users confined in the transmitter antenna beam.

Most of the researchers which have dealt with the uplink and downlink together in their researchers mainly consider the satellite transponder type in their models. It is known that there are two types of transponders [3], Regenerating and Bent-pipe transponders, the former that make reception and retransmission processes of the signal reached the satellite antenna in terms of decoding, demodulation, and information-processing actions before the encoding, modulation and amplification processes. Moreover, other actions could be added in this transponder, such as routing processes and beam switching between beams in mutli-spot beam antenna that will be explained in the next paragraph. While the Bent-pipe works as a repeater who limited to amplification for improvement in signal strength and retransmission activities.

An algorithm according to the beam type may also be built for certain types of researches that, for example, deal with accessibility or the studies that are not dealing with multiple accesses. There are mainly two types of antenna in terms of spot beam coverage [4], including the single beam satellite antenna and multi-spotbeam satellite antenna that cover several specified areas.

The chief reasons why there is an urge to establish the resource management in general as well as constructing the scheduling algorithm in a particular sense is claimed to be for the changes which might arise in the link quality encountered by each terminal. This may result from several sources, among which the mobility, weather conditions [5], jamming, congestion, etc. Another noticeable reason is a rise in the requirements as well as being able to maintain the QoS Level. A least reason would be twofold: primarily, there is a requirement to support high traffic requirements (eg. Bit rate) over the radio interface; moreover, the QoS demanded by the single applications which place new requirements on the resource management is supposed to be maintained thoroughly.

\subsection{Earth station(s) specifications}

The number of the stations on the earth had a major influence; it can decide the network layer used, performance matrices, resource allocation, etc.., rather than the station position, which affect the hop length; this will add another applicable performance matrix like error rates due to the position distance, accessibility, availability and handover matrices, in which all affects the Quality of Service (QoS). Moreover, the earth node could be either fixed or mobile; in case of mobility, like car, train and aircraft, several considerations should be added [6], such as Doppler shift, handover, shadowing, in addition to the difference in aspects related to accessibility and availability.

Though, the user(s) domain is needed to be considered as well; European Telecommunications Standards Institute (ETSI) [2] divides them into three network scenarios as follows:

- Core Network: represents the center to connect the routers in an area.

- Distribution Network: which works as an intermediate network connects the access network with the core network.

- Access Network: the edge network that provide services to the end users.

Moreover, the feedback channel can be also added to the algorithm or even this channel can be used as the main interest to build an algorithm. The feedback is used to identify the user QoS re- 
quirements, communication imperilments level, supported data, etc...; this channel is usually referred to as Channel state information (CSI).

\subsection{QoS requirements and layer specification}

A "Single layer approach" maintains that every case study is required to observe the OSI layer protocol; "Cross layer approach" is also referred to multi-layer protocols. The abovementioned approaches are supposed to maintain various aspects if they are intended to fulfill the goal of the building applicable wellformulated algorithm. The required aspects to be observed are briefed as follows.

At the physical layer, bandwidth or energy efficient algorithms regarding various modulation and encoding methods can be employed for boosting the Bit Error Rate (BER) as well as enhancing the performance of the power level when the distance difference between the satellite and station(s) became the control parameter, especially in mobility scenarios, or when the weather condition is poor; this can be illustrated as heavy rain which will possibly result in link bandwidth variations alongside a decrease in the obtainable capacity or a reduction in the carrier-to-noise and bit energy to noise ratios. Moreover, fading effect on the channel quality, delay spread, Doppler shift, restricted spectrum, path loss and the thermal noise are typically considered as some of the most notable phenomena which can be analyzed and simulated at the physical layers. [7] explores the potential bandwidth savings when using a combination of sharing spectrum on satellite transponders to enable bandwidth savings as well as developed error correction techniques. While the objective of [8] is to maximize the power and bandwidth efficiency in communication with small satellite terminals. Moreover, [9] dealt with a development of adaptive coding and modulation for different transmission schemes.

At the data link layer, bandwidth is needed to be guaranteed. This can be definitely attained through employing adequate bandwidthon-demand multiple access schemes as well as surveying the mechanisms interaction during several link constraints like the presence of jamming, blocking or fading of information. When it is said that the physical layer is required to provide a specific bandwidth to the upper layers, it is meant that there should be a bandwidth allocation scheme which is able to share the accessible bandwidth among various user terminals which have numerous traffic categories. [10] overviewed the bandwidth management issues in broadband multimedia satellite (BSM) in a hierarchical manner. While [11] presents a new approach for provisioning guaranteed bandwidth recovery in presence of link failures.

The network layer is required to distinguish the communication subnet topology and then to select a suitable paths through such a subnet. Therefore, the network layer handles the source-todestination delivering of the connection requests circuit and packet-switched networks. As declared, For the purpose of choosing the paths which impose the lowest congestion probability, there is a need to implement effective routing policies at this level.

The user mobility is necessary to be considered effectively when IP traffic management is taken into account. Henceforward, a prioritized management is required to be provided by the protocols of the network layer for the traffic which come from the users that occur in handover phases (such as in the presence of non-GEO satellites). Moreover, there is a need to sufficiently map mechanisms for IP-layer QoS provision to the MAC layer (RRM protocols); in addition to considering the protocol layering overhead, MAC queues, which are normally not in one-to-one correspondence to the former ones, supply the network layer queues with the service capacity. However, all the existing bandwidth at the transport layer is possible to be lodged by TCP connections that typically organize the traffic bulk needed to be transferred in the Internet. TCP traffic is naturally asymmetric in which the data flowing is in one direction whereas the acknowledgments flow in the reverse direction. This is normally translates into various bandwidth requirements respectively from the sender and the re- ceiver. It needs to be asserted that the TCP goodput is largely influenced by the bandwidth assignment and link quality. [12] uses network layer in their cross layer architecture proposed to increases the efficiency of the satellite resource utilization and minimizing the connection blocking and dropping probabilities for the physical channel variations. [13] give a solution to the limitation of the network specific environment that is TCP based and other upper-layer protocol performance by developing advanced Performance Enhancing Proxies (PEP) architecture.

Numerous traffic types (e.g., real and non-real time traffic) are required to ensure particular service level agreements at the application layer. In addition, for the purpose of adjusting the service priority adaptively, a monitoring feat is needed to be executed in association with the network layer. [14] presents a method which allows fine synchronization of satellite networks working at the application layer while maintaining the message exchange at the lowest amount.

However, the most recognized solutions for providing QoS in IP networks are provided by Internet Engineering Task Force (IETF) through Integrated Services (IntServ) [15], and Differentiated Services (DiffServ) [16].

IntServ and DiffServ both are able to supply the routers with queuing, scheduling and shaping that are some of the recognized QoS mechanisms which are applied in the router interfaces. Nonetheless, IntServ differ from DiffServ in the level of details employed by the classifiers. They also differ in their necessity of keeping the state information.

It is highlighted that the cross-layer methods are normally divided into two extensive categories, implicit and explicit cross-layer design. For implicit, it is declared that during the operation, there is no information transfer among various layers in certain algorithm is built in a specific layer without transferring among various layers, the interactions of the cross-layer are to be deliberated in case a joint optimization is intended during the design phase. In explicit design, for dynamic adaptations, there is a possibility of making the internal state of a protocol available to the protocols at various layers through using signaling interactions among nonneighboring levels of the protocol.

Consequently, it is to be admitted that the managing or scheduling process ought to be executed if the quality of service is intended to be boosted. Such a goal can be fulfilled through implementing appropriate variations in the employed performance indicators. What follows is a list of such indicators: Throughput, Resource Utilization, Total revenue, CNR, link availability, error rates, hop Delay, transmission Jitter, Call dropping rate, etc..

Seemingly, the error rates have been reported to be considered by the researchers as necessary matrices. In line with this, the mentioned error rates are typically being calculated through various types of calculations; these types include the Packet Error rate (PER), the Bit Error rate (BER), the Symbol Error rate (SER), and the Frame Error Rate (FER) in which video transmission is made use of.

As stated, definite kind of requirements should be provided by the QoS to assure the users. Such requirements can be listed when answering the some questions, how fast can the data be transmitted? How long does the receiver have to wait? How correct is the received data? And how much data is probably lost?

For this reason, several standardization groups provided standards for the quality of service in multimedia transmission, such as International Telecommunication Union (ITU), European Telecommunications Standards Institute (ETSI), Telecommunications Industry Association (TIA), and Universal Mobile Telecommunications System (UMTS). ITU recently concerned with the recommendations on performance of enhancements of TCP over satellite QoS architectures and performance, and reliable multicast Protocols for Satellites.

Broadband Satellite for Multimedia section of ETSI (ETSI/BSM) involved in developing air interface specifications for broadband communications rather than multicast architecture. TIA involved in developing air interface specification standard as well as ETSI, 
with other standards concerning IP Over Satellite Specification, Data Management Association (DAMA), and QoS Signaling. Because of the fact that specific requirements of QoS are commonly expected by the users from the satellite communication system, such requirements have been categorized by UTMS into four central categories. Indeed, they include Conversational services, Interactive services, Streaming services, and Background services. While ITU [17] categorize the services according to the time delay into four groups, Interactive, Responsive, Timely, and non-critical, and each service in a group could be error-tolerant or error-intolerant, Table 1 shows the groups with some application examples.

For conversational voice, the delay range from 0 to $150 \mathrm{~ms}$ was reported to be a preferred range; while the users are said not to feel there is no delay below $30 \mathrm{~ms}$, they would not sense any delays above $100 \mathrm{~ms}$ under two conditions: first, this happens if the echo cancellation is supplied. Secondly, it would be the case if distortions arise in the link. While the range from 150 to $400 \mathrm{~ms}$ : this is said to be an acceptable range; yet, there would be an increase in the degradation. The above $400 \mathrm{~ms}$ delay is claimed to be totally an improper range.

In videophone, it is supposed to transmit both video and audio Due to the fact that the human eyes can tolerate some information loss, it is then accepted to have packet loss to some extent. In addition, high performance video codecs are supposed to grant a satisfactory video quality with PLR up to about $1 \%$.

The network is used by interactive games for the purpose of interacting with the other systems or users. It is maintained that the requirements for interactive games are determined by the particular game involved with respect to the bandwidth and delay. A very large amount of data is exchanged through interactive games while they require pretty short delays; in this way, a delay of 200 $\mathrm{ms}$ is declared to be totally accepted.

Telnet (TELetype NETwork) is a network protocol which is being employed on the LAN network connections. To let a user's perception of interactivity, Telnet also requires a low delay.

Although voice messaging service resembles the conversational voice in the requirements for information loss, it is contrarily more tolerate to the delay; the reason for such a tolerance has been admitted to be the lack of direct conversation. Consequently, its main duties include defining the delay to be tolerated between the users, allotting a command to repeat a voice message, and starting the audio in an actual way. Although no accurate data is available concerning this type of service, it is acceptable for this application to have a delay of roughly a few seconds.

Having requested a Web page, the foremost performance factor is said to be visualizing the response time. Therefore, a value of 2-4 $\mathrm{s}$ per page is to be recommended although a reduction down to a target of $0.5 \mathrm{~s}$ is still appropriate.

Streaming services category is mostly recognized to be unidirectional while it is having a high uninterrupted utilization (few idle/silent periods); in addition, within a flow it has a low time variation between the information entities. Because of the fact that the stream is typically associated at the destination, no severe restriction is needed for the delay as well as the delay variation. Besides, no severe upper limit is required for the packet loss rate.

Major QoS characteristics for real-time streams encompass unidirectional continuous stream as well as preserving time relation (variation) between the information entities of the stream. At last, this communication scheme is generally intended is to support real-time streaming services which have uninterrupted unidirectional data flows. The three mostly used applications are audio streaming, one-way video, and still image. In contrast with the conventional telephony, the audio streaming yields a better quality; for this reason, it can be then concluded that the packet loss requirements are tighter accordingly. Nonetheless, no conversational elements are engaged while the delay requirements will be also relaxed.
Table 1: Services Categorization

\begin{tabular}{|c|c|c|c|c|}
\hline Services & \begin{tabular}{c|c} 
Delay \\
variation
\end{tabular} & $\begin{array}{l}\text { tolerable } \\
\text { Error rate }\end{array}$ & $\begin{array}{l}\text { Data } \\
\text { rate }\end{array}$ & One way delay \\
\hline $\begin{array}{c}\text { Bulk data } \\
\text { transfer/retrieval }\end{array}$ & N.A. & $\begin{array}{c}\text { Error- } \\
\text { intolerant }\end{array}$ & & $\begin{array}{c}<15 \text { s/page (Pre- } \\
\text { ferred) } \\
<60 \mathrm{~s} / \text { page (Ac- } \\
\text { ceptable) }\end{array}$ \\
\hline $\begin{array}{l}\text { Low priority } \\
\text { data } \\
\text { transactions }\end{array}$ & N.A. & $\begin{array}{c}\text { Error- } \\
\text { intolerant }\end{array}$ & & $<30 \mathrm{~s}$ \\
\hline $\begin{array}{l}\text { Web browsing, } \\
\text { Email, and } \\
\text { transactions } \\
\text { information }\end{array}$ & N.A. & $\begin{array}{c}\text { Error- } \\
\text { intolerant }\end{array}$ & & $\begin{array}{c}<2 \text { s/page (Pre- } \\
\text { ferred) } \\
<4 \text { s/page (Ac- } \\
\text { ceptable) }\end{array}$ \\
\hline $\begin{array}{l}\text { Interactive } \\
\text { games and } \\
\text { Telnet }\end{array}$ & N.A. & $\begin{array}{c}\text { Error- } \\
\text { intolerant }\end{array}$ & & $<200 \mathrm{~ms}$ \\
\hline Fax & N.A. & $\mathrm{BER}<10^{-6}$ & & \\
\hline Streaming audio & $<1 \mathrm{~ms}$ & PLR $<1 \%$ & $\begin{array}{c}16-128 \\
\text { kbps }\end{array}$ & $<10 \mathrm{~s}$ \\
\hline $\begin{array}{l}\text { Voice messag- } \\
\quad \text { ing }\end{array}$ & $<1 \mathrm{~ms}$ & PLR $<3 \%$ & $\begin{array}{l}4-32 \\
\text { kbps }\end{array}$ & $\begin{array}{c}<1 \mathrm{~s} \text { for playback } \\
<2 \mathrm{~s} \text { for record }\end{array}$ \\
\hline $\begin{array}{l}\text { Conversational } \\
\text { voice }\end{array}$ & $<1 \mathrm{~ms}$ & PLR $<3 \%$ & $\begin{array}{l}4-64 \\
\text { kbps }\end{array}$ & $\begin{array}{c}<150 \mathrm{~ms} \text { (pre- } \\
\text { ferred) } \\
<400 \mathrm{~ms} \text { (Limit) }\end{array}$ \\
\hline Video phone & & PLR $<1 \%$ & $\begin{array}{c}16-384 \\
\text { kbps }\end{array}$ & $\begin{array}{c}<150 \mathrm{~ms} \text { (pre- } \\
\text { ferred) } \\
<400 \mathrm{~ms} \text { (Limit) }\end{array}$ \\
\hline
\end{tabular}

Moreover, the lack of conversational elements can be enumerated as the one-way video' prominent characteristic so that in which the delay requirements are not very severe. However, when still images are considered, it can be declared that human eyes can tolerate the information loss; yet, great disturbances can be cause by single bit errors in still image formats. For such reasons, zero errors are commonly anticipated to arise during the broadcast of still images. In addition, the delay requirements are low, as well.

Background services are customarily used once the end-user which is normally a computer is involved in sending and receiving the data files in background. The point to be highlighted is that this kind of service is indeed a classical data communication scheme in which the destination never expects the data during a determined limit. Yet, the only requirement for applying this service is error-free delivered information. The fact is that the data is totally useless if received too late; henceforth, a delay constraint will be anticipated. Some of these applications can be exemplified as Fax, and some low priority transaction services.

Although fax is not generally measured as a real-time communication, its transmission is yet anticipated to last less than 30 seconds. While in low priority transaction services such as e-mail is from server to server, Short Message Service (SMS) that has a tolerable delivery delay of about 30 seconds, and databases download as well as measurement records.

\subsection{Scheduling techniques}

QoS levels provided by a network can be significantly influenced by the nature of scheduling mechanisms which are used on network links. However, after considering parameters and specifications mentioned before, it is the time now to identify the buffer characteristic. This is done by identifying the buffer size needed in packets or bits, prior to assigning its type. There are numerous buffer types but the most used ones are the Drop-Tail and Random 
Early Detection (RED). The former is the simplest one because the dropping of packets arrived occurs when the buffer is filled. While the procedure of the latter is to start dropping the packets linearly as the minimum specified threshold of buffer occupation reached, while all the arrived packets from a source will be dropped when the maximum specified threshold of buffer occupation reached (usually when the buffer is about to be filled) therefore an average number of packets in the buffer is maintained.

As a matter of fact, arbitrating between the packets which are prepared to be transmitted on the link is asserted to be the scheduler's chief function. Hence, definite performance measures can be attained by considering both the scheduling algorithm alongside the traffic characteristics of the flows multiplexed on the link. In order to provide end-to-end QoS guarantees, these can be employed by the network. The commonly used scheduling techniques are discussed in the following paragraphs.

First in First out (FIFO) is proclaimed to be the simplest scheduling technique at present that is said to be also the most extensively used technique in the Internet. In FIFO which can be also taken for "First Come, First Served" (FCFS), packets are served in keeping with the order by which they arrive. Nonetheless, no guarantees are granted to the end-users by this scheduling policy. [18] investigated the channel assignment scheme for handover call process in LEO satellite using FIFO as one of the scheduling techniques.

Fixed Priority Mechanisms attempt to provide the lowermost likely delay for the uppermost priority class between two or more classes. In this mechanism, the link multiplexer upholds a distinct queue for each priority. Prior to sending the data for the next class, the scheduler sends them from the utmost priority class. In case all the higher priority queues are empty, a packet is served then in a lower priority queue. Fixed priority schedulers are considered as simple as the FIFO scheduler that has the additional complexity of holding queues. This is the case because each queue is served in an FIFO manner. The discussed scheduling technique provides service differentiation; nevertheless, lower priority classes should not be starved.

Weighted Round Robin (WRR) is also a technique for guaranteeing a minimum allocation and distribution; it allows a weighted access to the obtainable bandwidth to each class. According to the weights, each class in a round-robin manner is serviced by the scheduler. Moreover, the vacant capacity is dispersed to the other classes according to their weights in case one or more classes do not use their full allocation. A lesser effective delay is obtained by a class through granting it with a higher weighting than the traffic level it is transmitting. [19] developed an adaptive algorithm that optimizes the bandwidth utilization by managing the weights of a WRR scheduler for various traffic classes.

Class-Based Queuing (CBQ) [20] generally refers to any mechanism which is based on the class. While every class is accompanied with a quantity of the link bandwidth, assuring that this bandwidth of the traffic belongs to the class is said to be a goal of this technique. In case there is a surplus bandwidth, it would be thus shared fairly among the other classes. The similar scheduling policy is not to be necessarily employed at all of the levels of a link sharing hierarchy.

Weighted Fair Queuing (WFQ) along with its variants, through employing a grouping of weighting and timing information for arbitrating which queue to be served, is proposed for distributing the available bandwidth over several weighted classes. As such, the weighting controls the ratio of the bandwidth distribution efficiently between the classes which are at the moment under congestion. [21] implemented WFQ into LEO satellite link system to provide end-to-end delay guarantee with high throughput.

The Earliest Deadline First (EDF) is recommended as a dynamic priority scheduler that owns innumerable priorities. In this way, each packet's priority is granted by its deadline. The Earliest Deadline First technique would be optimal [22] in case under any scheduling disciplines, a number of tasks are schedulable. Similarly, the set is also schedulable under EDF. At last, it should be admitted that the providing end-to-end delay guarantees will be allowed by EDF scheduling alongside per-class traffic shaping. [23] evaluated the EDF scheduling technique for deadline-aware scheduling application in data broadcast server design for requests with certain response deadlines.

Shortest Window First (SWF) gives priority to packets from the upper layers instructions with the slow sessions. SWF schedules with admission of packets into the queue according to packets' source window size. The Packets arrived with smaller source window sizes will be the slower packets. The packets that based on window size guarantee SWF is sensitive to packets that suffer from timeout events. It also permits packets to initially open their congestion windows rapidly. The Smallest Sequence Number First (SSF) scheduler is similar to the SWF scheduler, except that in SSF scheduling, the sequence numbers of packets determine the priority class, i.e. the sequence number is the indicator of slowness. [24] designed buffer management strategies for enhancing the performance of transfer control protocol using SWF and SSF scheduling techniques.

\section{Conclusion}

Recently, building efficient scheduling algorithms gained the utmost necessity for satellite communication link designers according to the level advance technology and the wide applications intended by billions of users. The paper presented the optimal approach with guidance for building such efficient algorithms; it presents the effective parameters that the designers should take into consideration, specifically, the satellite node parameters considerations such as the satellite height, mobility, and type according to connections, equipment, and functions. As well as earth station node parameters in terms of number of users, mobility, position, connection and domain types.

After specifying the abovementioned parameters it is recommended to subsequently identify the communication layer associated with user requirements and applications. This will lead the designer to properly identify other chief specifications of efficient scheduling algorithm, as well as identifying the problems with a funnel to proper solutions even before building the algorithm. These specifications, such as user requirements, service limitations, protocol stacks, performance matrices, control factors, connection environment and obstacles, and link specifications, are identified and discussed with their characteristics to represent the proper approach for building efficient scheduling technique in satellite communications. Upon starting the design of such algorithm, the paper had introduced the efficient scheduling techniques that the researchers used to build their scheduling algorithms associated with appropriate applications. The developed guidance is recommended to be used for building efficient scheduling algorithm that will provide proper solutions to the satellite communications impairments.

\section{References}

[1] F. Vieira, M. A. V. Castro, and L. Jiang, "Datacast transmission architecture for DVB-S2 systems in railway scenarios," in Signal Processing for Space Communications, 2008. SPSC 2008. 10th International Workshop on, 2008, pp. 1-5.

[2] E. T. S. I. (ETSI), "Satellite Earth Stations and Systems (SES) Broadband Satellite Multimedia (BSM); Services and architectures," in TR 101984 ed: European Telecommunications Standards Institute, 2007.

[3] W. Guo, X.-Y. Gong, X.-Y. Xu, L. He-Guang, C.-D. Xu, and Y.-H. $\mathrm{Du}$, "A transponder system dedicating for the on-orbit calibration of China's new-generation satellite altimeter and scatterometer," in IEEE CIE International Conference on Radar, 2011, pp. 22-25.

[4] Y. Hwang and Y. H. Choung, "Satellite Antennas," in Communications Handbook, J. D. Gibson, Ed., ed: CRC PressINC, 2002.

[5] A. M. Al-Saegh, A. Sali, J. S. Mandeep and F. Pérez Fontán, "Channel Measurements, Characterization, and Modeling for Land Mobile 
Satellite Terminals in Tropical Regions at Ku-band,", IEEE Transactions on Vehicular Technology, vol. 66, no. 2, pp. 897-911, 2017.

[6] I. T. Union, "Propagation data required for the design of Earth-space land mobile telecommunication systems," Recommendation ITU-R P.681-7, ed: International Telecommunication Union, 2009.

[7] C. Agne, M. B. Cornell, M. Dale, R. Kearns, and F. Lee, "Sharedspectrum bandwidth efficient satellite communications," in Military Communications Conference, Milcom 2010, 2010, pp. 341-346.

[8] A. Tomlinson, A. Ambroze, and G. Wade, "Power and bandwidth efficient modulation and coding for small satellite communication terminals," in IEEE International Conference on Communications ICC 2002, pp. 2943-2946 vol.5

[9] I. B. Djordjevic and G. T. Djordjevic, "Adaptive modulation and coding for generalized fading channels," in 9th International Conference on Telecommunication in Modern Satellite, Cable, and Broadcasting Services, TELSIKS '09., 2009, pp. 418-422.

[10] F. Shaodong, L. Guangxia, W. Fan, and F. Qi, "Research Issues on Bandwidth Management in Broadband Multimedia Satellite Communication System," in Recent Advances in Computer Science and Information Engineering. vol. 127, Z. Qian, L. Cao, W. Su, T. Wang, and H. Yang, Eds., ed: Springer Berlin Heidelberg, 2012, pp. 717-722.

[11] W. Lau, S. Jha, and S. Banerjee, "Efficient bandwidth guaranteed restoration algorithms for multicast connections," NETWORKING 2005. Networking Technologies, Services, and Protocols; Performance of Computer and Communication Networks; Mobile and Wireless Communications Systems, pp. 237-243, 2005.

[12] J. D. Del Rio, O. D. R. Herrero, and M. F. Veiga, "A dynamic crosslayer architecture for QoS provisioning of multimedia services on allIP based adaptive satellite networks," in International Workshop on Satellite and Space Communications, IWSSC 2009. 2009, pp. 364-368.

[13] J. Kim, D. H. Wang, D. G. Oh, and B. Park, "A design methodology of advanced-PEPs architecture for TCP satellite connection and bandwidth management," International Journal of Multimedia and Ubiquitous Engineering, vol. 7, pp. 141-148, 2012.

[14] A. Marco, R. Casas, J. L. Sevillano, V. Coarasa, J. L. Falco, and M. S. Obaidat, "Multi-Hop Synchronization at the Application Layer of Wireless and Satellite Networks," in Global Telecommunications Conference, IEEE GLOBECOM 2008. IEEE, 2008, pp. 1-5.

[15] R. Braden, Clark, D., Shenker, S. , "Integrated Services in the Internet Architecture: an Overview," RFC Editor, IETF1994.

[16] S. Blake, Black, D., Carlson, M., Davis, E., Wang, Z., Weiss, W., "An architecture for differentiated services," IETF1998

[17] I. T. U. ITU, "End-user multimedia QoS categories.," ed: International Telecommunication Union - Recommandation G.1010, 2001.

[18] Y. Jia and Z. Peng, "Channel reservation and queuing in Low Earth Orbit mobile satellite system," in International Congress on UltraModern Telecommunications and Control Systems and Workshops (ICUMT), 2010, pp. 734-738.

[19] E. Rendon-Morales, J. Mata-Díaz, J. Alins, J. Muñoz, and O. Esparza, "Adaptive Packet Scheduling for the Support of QoS over DVB-S2 Satellite Systems," in Wired/Wireless Internet Communications. vol. 6649, X. Masip-Bruin, D. Verchere, V. Tsaoussidis, and M. Yannuzzi, Eds., ed: Springer Berlin Heidelberg, 2011, pp. 15-26.

[20] S. Floyd and V. Jacobson, "Link-sharing and resource management models for packet networks," IEEE/ACM Transactions on Networking, vol. 3, pp. 365-386, 1995.

[21] H. Qijie, Y. Boon Sain, and K. Peng-Yong, "An enhanced QoS routing algorithm for provision of end-to-end delay guarantee in low earth orbit satellite networks," in IEEE Wireless Communications and Networking Conference, 2005, pp. 1485-1490 Vol. 3

[22] L. Georgiadis, R. Guerin, and A. Parekh, "Optimal multiplexing on a single link: delay and buffer requirements," IEEE Transactions on Information Theory, vol. 43, pp. 1518-1535, 1997.

[23] C. Weiwei and D. Aksoy, "Approximation issues for soft QoS support in large-scale broadcast-based networks," in IEEE 60th Vehicular Technology Conference, VTC2004-Fall, 2004, pp. 4041-4045 Vol. 6.

[24] S. Jain and E. Modiano, "Buffer management schemes for enhanced TCP performance over satellite links." In IEEE Military Communications Conference, MILCOM 2005., pp. 1672-1678. IEEE, 2005. 